\title{
New Mechanisms for Lack of Equipartition of Energy
}

\author{
Àlex Haro* and Rafael de la Llave ${ }^{\dagger}$ \\ Department of Mathematics, University of Texas at Austin, Austin, Texas 78712
}

(Received 14 February 2000)

\begin{abstract}
We describe several mechanisms that prevent equipartition of energy in mechanical systems. In certain regimes, we present a quantitative prediction of the relative abundance of orbits exhibiting these mechanisms. This quantitative prediction is confirmed in numerical experiments.
\end{abstract}

PACS numbers: 05.45.Xt, 05.45.Pq, 05.45.Ra, 45.10.Hj

One of the basic problems of statistical mechanics [1] is to decide its range of applicability, in particular, the validity of the equipartition of energy. Deciding what are the boundaries of applicability of statistical mechanics has become one of the fundamental problems not only for the foundations but, indeed, for the applications.

Very early it was realized through numerical experiments [2] that many systems which indeed allow statistical mechanics descriptions in certain regimes do not behave statistically in others. The original findings of [2] that energy does not spread in certain regimes have been confirmed and extended in several places (see, for example, [3] and [4] for a history).

The only mechanism proposed to explain lack of equipartition of energy is, to the best of our knowledge, based on Kolmogorov-Arnold-Moser theory (KAM). This theory shows that if a system is a sufficiently small perturbation of an integrable system, some of the quasiperiodic motions characteristic of the integrable system persist. Indeed, the initial conditions of quasiperiodic motions cover a set of positive measure of phase space. Hence, they lead to the lack of thermodynamic behavior. (See [5] for a review of the relevance of perturbation theory in statistical mechanics and [6] for a KAM theorem taylored for these systems.)

The geometric interpretation of a quasiperiodic orbit is an invariant torus [7]. This geometric interpretation will be very important in subsequent arguments; hence, we will use the geometric name invariant tori for these objects.

In this Letter, we suggest other mechanisms for lack of equipartition of energy. As we show in some numerical experiments, they are easily observable and very quantifiable.

The mechanism is based on the observation that perturbations of integrable systems not only destroy the periodic motions present in the original systems but also generate new ones. This is the well-known phenomenon of islands, which are quite apparent in Fig. 1.

The main point of this Letter is that in many mechanical systems these islands-or their higher dimensional analogs (which we call secondary tori; see below) - are much more relevant for statistical mechanics than KAM tori. More precisely, we argue that the measure of the secondary tori is much larger than that of primary KAM tori.
Since measure translates into probability in statistical mechanics, secondary tori are statistically more relevant than KAM tori.

Our argument leads to precise quantitative estimates of the measures of these tori. We will present numerical evidence, which matches extraordinarily well our quantitative prediction.

The concept of secondary tori, central to our argument, can be easily grasped in the example of the well-known standard map. Standard KAM theory shows the existence of invariant circles which are not contractible to a point. (In Fig. 1, these are the circles that are roughly horizontal and go across the angle coordinate $x$.) A prominent feature of the phase portrait is the islands. They contain invariant circles that, in contrast to the KAM tori, can be contracted to a point. Note that these contractible tori are not present in the integrable system. In classical mechanics books, the contractible circles are called librating and the noncontractible ones rotating.

In general, given a system whose phase space is $\mathbf{T}^{d} \times$ $\mathbf{R}^{d}$, we say that a $d$-dimensional invariant torus with a quasiperiodic motion on it is a secondary torus when it is contractible to a lower dimensional torus.

In [8] it is shown that in quasi-integrable systems, one should find quite often secondary tori generated by resonances.

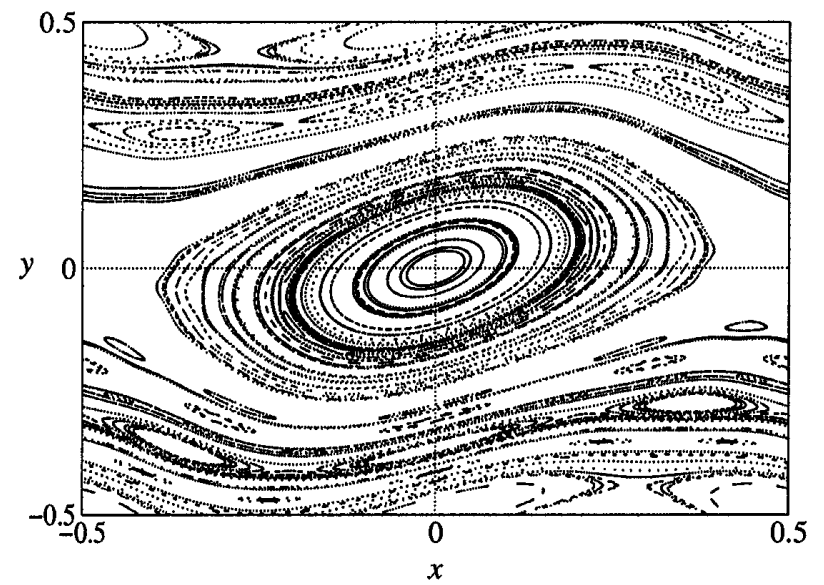

FIG. 1. Primary and secondary circles in the standard map, a model of symplectic map in $\mathbf{T} \times \mathbf{R}$. Secondary circles lie in islands surrounding elliptic periodic orbits. 
We introduce an observable that can find a difference between KAM tori and secondary tori. Besides being able to distinguish between KAM tori and secondary tori, the observable is easy to measure in numerical experiments and we can develop a theory of its dependence on the number of degrees of freedom.

We will describe our observable for maps. For continuous time systems, it suffices to take the time-1 map. We will consider systems whose phase space is angle-action $\left(\mathbf{T}^{d} \times \mathbf{R}^{d}\right)$. If the phase space is $\mathbf{R}^{d} \times \mathbf{R}^{d}$, we take symplectic polar coordinates.

We say that the $i$ th coordinate of (the lift of) an orbit $z^{n}=\left(x^{n}, y^{n}\right)=F^{n}(z)$ is resonant if there exists $p \in$ $\mathbf{Z}, q \in \mathbf{N}$ such that the sequence

$$
\left|x_{i}^{n}-x_{i}^{0}-n p / q\right|
$$

is bounded. Fixed a positive integer $N$, we say that the orbit is a class-k orbit if $k$ of its coordinates are resonant with $|q| \leq N$.

Roughly speaking, saying that the $i$ th coordinate is resonant means that the $i$ th coordinate is trapped near a $p / q$-periodic orbit of the uncoupled system. (In the classical pictures of maps, these are orbits in the islands near a periodic orbit.) Note that if the resonance condition is satisfied for $q=1$, then the orbit is librating.

The feature of this observable that we want to emphasize is that KAM tori have class 0 . Hence, objects of nonzero class cannot be KAM tori. We are going to argue that class zero is rare among invariant tori. Therefore, we will conclude that, a fortiori, KAM tori are also rare.

We have studied systems which are representative of the physical situation covered by statistical mechanics: identical systems (oscillators) with local couplings. The following assertions summarize our findings (heuristic arguments supported by numerical evidence, but so far, no rigorous proofs).

The first assertion concerns the measure of orbits of a certain class.

Assertion 1: For a quasi-integrable system of d coupled oscillators, provided that the couplings are such that the anharmonic forces are larger than the forces of the local couplings, the measure occupied by the class-k tori in a region of phase space is approximately

$$
p(k)=\left(\begin{array}{l}
d \\
k
\end{array}\right) a^{d-k} b^{k},
$$

where $a, b$ depend on the coupling constants and the region of phase space.

We now assert that the coefficients $a$ and $b$ are effects mainly of the coupling constants.

Assertion 2: The coefficients $a, b$ in Assertion 1 are almost independent of the number of oscillators. They depend on the coupling constants.

Note that (1) implies that class zero tori are a fraction $[a /(a+b)]^{d}$ of the total number of tori, which is small when $d$ is large. KAM tori are, of course, a smaller fraction.

An illuminating example that gives the idea why (1) could be true is to consider a system of $d$ uncoupled standard maps. If each of the standard maps is moving in an invariant circle, the whole system is moving in a torus which is the Cartesian product of the circles in each standard map. Note that the class of this torus is precisely the number of secondary circles of the components.

If the standard map has a fraction of primary circles $a$ and a fraction $b$ of secondary circles, then, the class of these products will satisfy (1) as can be seen from elementary combinatorics (recall that the measure of the Cartesian product is the product of the measures).

More generally, we argue on physical grounds that whether an oscillator is librating or rotating is almost independent of what oscillators far away are doing. Therefore, the number of librating coordinates should be well represented by a sum of independent variables, hence a binomial distribution.

Note that the independence of far away oscillators is not to be expected to be valid always. For example, having a very strong local coupling would lead to the existence of waves, which could cause long range influence. A rough criterion for the range of validity of our Assertion 1 is that the local anharmonic forces should be stronger than the local coupling.

The justification of Assertion 2 comes from the fact that the phenomena that give rise to the classes are local. Hence, they should depend only on the local properties.

The law (1) suggests several natural issues, which we will not pursue here: (i) characterize the limits of validity in terms of the sizes of the couplings and the number of degrees of freedom, (ii) study the corrections for small (but finite) couplings and finite (but large) number of degrees of freedom, and (iii) study the possibility of existence of secondary tori for large couplings.

For issues (i) and (ii) we believe that the answer should be similar to those for the central limit theorem for weakly dependent variables. For (iii) we point out that [9] shows that there are secondary tori for arbitrarily large values of the coupling, whereas [10] shows that there are no KAM tori that are graphs for $\alpha+4 \beta \geq 2$ for the coupled standard maps (CSM) model.

Since resonances, the only proposed mechanism for the destruction of KAM tori, generate secondary tori, we think it is reasonable that secondary tori are relevant for statistical mechanics even for couplings for which KAM tori do not exist.

We have verified the assertions in several models. These models have been chosen to be representative of those appearing in statistical mechanics. Of course, the main strength of statistical mechanics is that its predictions are independent of the details of the model. Hence, we expect that similar agreement with the prediction will hold for a variety of models. 
The main model, henceforth referred to as $\mathrm{NCO}$ (from neighboring coupled oscillators), describes $d$ anharmonic oscillators with a local coupling. This model is indeed a canonical model of all physical processes in which there are nonlinear oscillations and couplings. For example, it is discussed in $[11,12]$ and it has an extensive literature (see [13-15]).

The model is described by a Hamiltonian in $\mathbf{R}^{d} \times \mathbf{R}^{d}$ :

$$
\begin{aligned}
H(x, y)= & \frac{1}{2} \sum_{i=1}^{d}\left(y_{i}^{2}+x_{i}^{2}\right)+\sum_{i=1}^{d} V_{1}\left(x_{i}\right) \\
& +\sum_{i=1}^{d} V_{2}\left(x_{i+1}-x_{i}\right),
\end{aligned}
$$

where $V_{1}(x)=\frac{\alpha}{4} x^{4}, V_{2}(x)=\frac{\beta}{2} x^{2}+\frac{\gamma}{3} x^{3}$, and $\alpha$ and the coupling constants $\beta, \gamma$ are positive parameters.

A discrete time model is the coupled standard maps, a symplectic map in $\mathbf{T}^{d} \times \mathbf{R}^{d}$ given by

$$
\begin{aligned}
& \bar{x}_{i}=x_{i}+\bar{y}_{i} \quad(\bmod 1), \\
& \bar{y}_{i}=y_{i}-V_{1}^{\prime}\left(x_{i}\right)+\left[V_{2}^{\prime}\left(x_{i+1}-x_{i}\right)-V_{2}^{\prime}\left(x_{i}-x_{i-1}\right)\right],
\end{aligned}
$$

where $V_{1}(x)=-\frac{\alpha}{4 \pi^{2}} \cos (2 \pi x), V_{2}(x)=-\frac{\beta}{4 \pi^{2}} \cos (2 \pi x)$ $(i=1 \div d$ and we identify $d=0$ ).

As the local anharmonic forces should be stronger than the local couplings, this amounts to $\alpha \gg 2 \beta$ for the CSM and $1+\alpha \gg 4 \beta+6 \gamma$ for the NCO.

For fixed values of the couplings we have chosen points of the phase space at random and followed their trajectories. In order to decide whether an orbit is in an invariant torus, we have computed the Lyapunov exponent. Those orbits with a small empirical Lyapunov exponent are taken as lying on an invariant torus. This is based on the fact that the only zero-Lyapunov objects with positive measure that have been proposed in the literature are invariant tori. For systems of large numbers of degrees of freedom, other criteria seem impracticable.

We have verified that the results are independent from several numerical choices such as the cutoff for rejection of a Lyapunov exponent, the numbers used to decide that the orbit was at a bounded distance from a rational, and the length of the orbits. For the systems we have considered, orbits seem to be easily classifiable in two categories: those whose empirical Lyapunov exponent grows fast and another one in which the Lyapunov exponent is very small (quantitative explorations of this issue are in [16] and some rigorous progress in [17]).

We have taken 32768 points at random. For the CSM model the initial points satisfy $\left|y_{i}\right|<\frac{1}{2}$, and for the NCO model $\left|x_{i}\right|<\frac{1}{2}$ and $y_{i}=0$ (zero velocity). The number $N$ that we have used in the results reported below is 7 , even if we have done runs with other numbers. The values of $a, b$ change with $N$, but the law (1) remains valid.

To integrate the NCO model, we have used the $S B^{3} A$ integrator described in [18], which is of order 6 and whose step is a symplectic map. As it is getting well accepted,

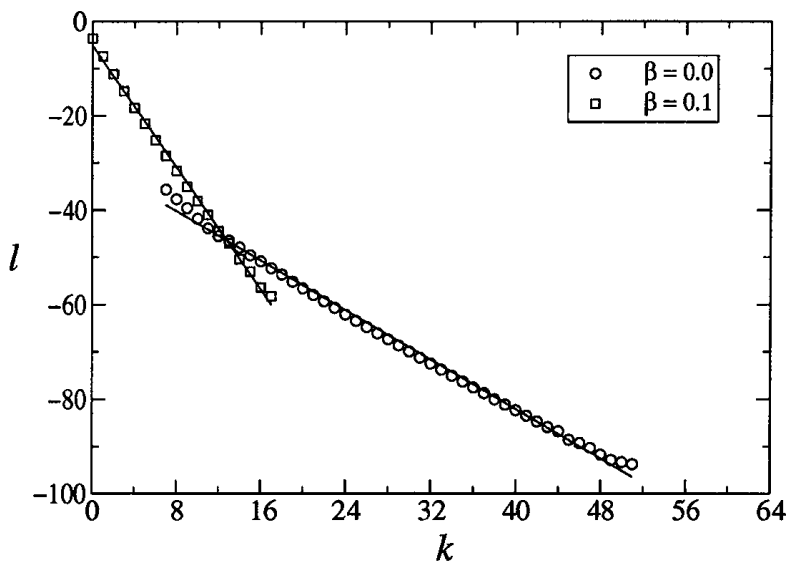

FIG. 2. Assertion 1: Verification of (2) for the NCO model with $d=128, \alpha=1, \gamma=0.1$.

to study statistical properties, it is convenient to rely on symplectic integrators.

The results are depicted in several figures.

Notice that Assertion 1 can be formulated as

$$
l(k):=\log \left[p(k) /\left(\begin{array}{l}
d \\
k
\end{array}\right)\right]=d \log a+k \log (b / a) .
$$

Then, we plot $l$ against $k$, and the assertion is equivalent to obtaining a straight line.

Some representative cases are included in Figs. 2 and 3. In Fig. 4 we have verified (2) for different degrees of freedom. Assertion 2 amounts to these lines being parallel. Note that $p(k)$ itself is almost a Gaussian centered in $b d /(a+b)$ and, therefore, away from the center, the probabilities of the points are very small so that the edges of the straight line correspond to a small number of observations. In Fig. 5, we have $a, b$ for different couplings and different numbers of degrees of freedom.

To fit the parameters of the straight lines, we have used the routine medfit from [19], which has the advantage over least squares that it is less sensitive to outliers.

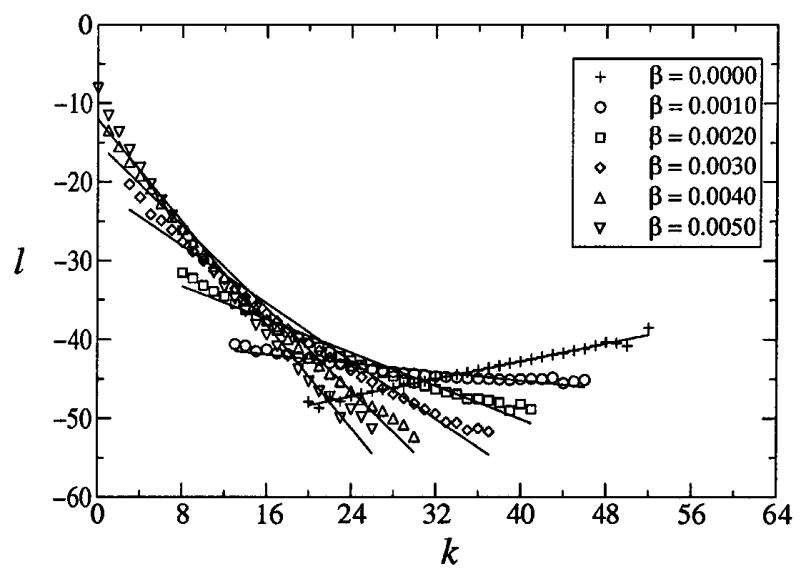

FIG. 3. Assertion 1: Verification of (2) for the CSM model with $d=64, \alpha=0.05$. 


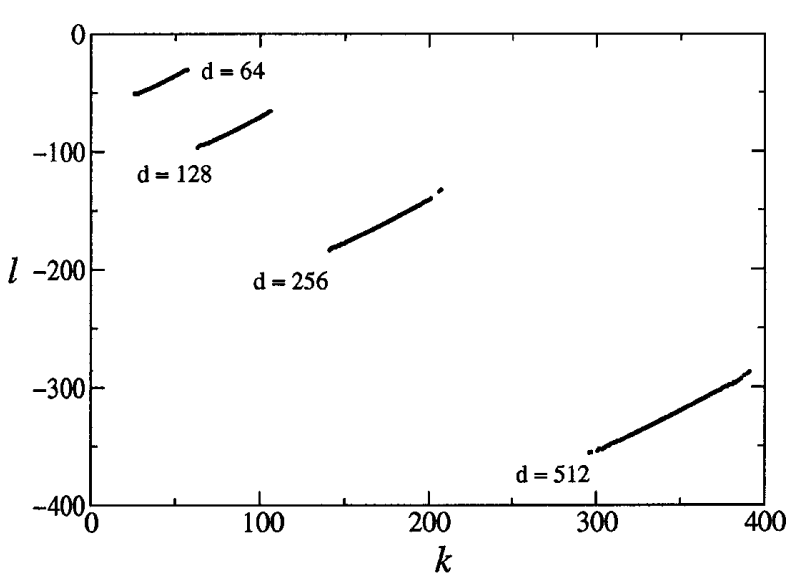

FIG. 4. Assertions 1 and 2: Verification of (2) for the CSM model with $\alpha=0.1, \beta=0.01$ and different degrees of freedom.

Even if the number of degrees of freedom increases by a factor of 8 and $\beta$ changes substantially so that $a$ and $b$ change by a factor of 2 , the values of $a$ and $b$ for the same $\beta$ are within $1 \%$ of each other according to Assertion 2 .

In summary, we have identified new mechanisms for lack of equipartition in mechanical systems, provided an easy way to measure them, and given quantitative predictions of their abundance. In particular, they are much more abundant than mechanisms previously considered. These predictions have been confirmed with very good accuracy in numerical experiments.

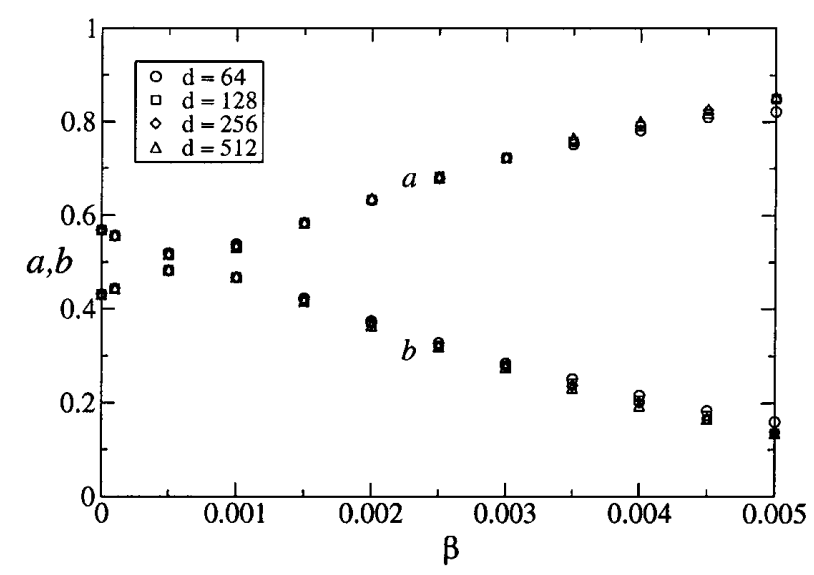

FIG. 5. Assertion 2: Estimated values of $a$ and $b$ for different degrees of freedom and coupling parameter $\beta$ for the CSM model with $\alpha=0.05$.
A.H. has been sponsored by the Fulbright Program and the Generalitat de Catalunya (Spain), and supported by Grants No. 94-0215 (DGICYT-Spain), No. ERBCHRXCT-940460 (EC), and No. 1998SGR00042 (CIRIT-Catalunya). The work of R. L. is partially supported by NSF grants.

*Permanent address: Departament de Matemàtica Aplicada i Anàlisi, Universitat de Barcelona, Gran Via 585, 08007 Barcelona, Spain.

Email address haro@math.utexas.edu

†Email address: llave@math.utexas.edu

[1] R. Balescu, Equilibrium and Nonequilibrium Statistical Mechanics (Wiley-Interscience, New York, 1975).

[2] E. Fermi, J. Pasta, S. Ulam, and M. Tsingou, "Studies of Nonlinear Problems I," Los Alamos Report No. LA-1940, 1955; E. Fermi, Collected Papers (University of Chicago Press, Chicago, 1965), Vol. II, p. 978.

[3] B. V. Chirikov, Phys. Rep. 52, 264 (1979).

[4] J. Ford, Phys. Rep. 213, 271 (1992).

[5] A. S. Wightman, Perspectives in Statistical Physics (NorthHolland, Amsterdam, 1981), pp. 343-363.

[6] C. E. Wayne, Commun. Math. Phys. 96, 311 (1984); 96, 331 (1984).

[7] V.I. Arnol'd, Ordinary Differential Equations (SpringerVerlag, Berlin, 1992).

[8] R. de la Llave and C. E. Wayne, report, 1989 (unpublished).

[9] P. Duarte, Ann. Inst. Henri Poincaré Anal. Non Linéaire 11, 359 (1994).

[10] A. Haro, Nonlinearity 12, 1299 (1999).

[11] R.P. Feynman, Statistical Mechanics (Perseus Books, Reading, MA, 1998).

[12] M. Born and K. Huang, Dynamical Theory of Crystal Lattices (Oxford University Press, New York, 1995).

[13] L. A. Bunimovich, Physica (Amsterdam) 103D, 1 (1997).

[14] N. Kopell and G. B. Ermentrout, SIAM J. Appl. Math. 50, 1014 (1990).

[15] G. Benettin, L. Galgani, and A. Giorgill, Nuovo Cimento Soc. Ital. Fis. 89B, 89-103 (1985); 89B, 103-119 (1985).

[16] S. Isola, A. Politi, S. Ruffo, and A. Torcini, Phys. Lett. A 143, 365 (1990).

[17] J. P. Eckmann and C.E. Wayne, J. Stat. Phys. 50, 853 (1988).

[18] R. I. McLachlan, SIAM J. Sci. Comput. 16, 151 (1995).

[19] W. H. Press, S. A. Teukolsky, W. T. Vetterling, and B.P. Flannery, Numerical Recipes in $C$ (Cambridge University Press, Cambridge, England, 1992), 2nd ed. 